\title{
piRNA-directed cleavage of meiotic transcripts regulates spermatogenesis
}

\author{
Wee Siong Sho Goh, ${ }^{1,2,5}$ Ilaria Falciatori, ${ }^{1,2,3}$ Oliver H. Tam, ${ }^{2}$ Ralph Burgess, ${ }^{1,2}$ Oliver Meikar, ${ }^{4}$ \\ Noora Kotaja, ${ }^{4}$ Molly Hammell, ${ }^{2}$ and Gregory J. Hannon ${ }^{1,2,3}$ \\ ${ }^{1}$ Howard Hughes Medical Institute, ${ }^{2}$ Watson School of Biological Sciences, Cold Spring Harbor, New York 11724, USA; ${ }^{3}$ Cancer \\ Research UK Cambridge Institute, University of Cambridge, Cambridge CB2 ORE, UK; ${ }^{4}$ Institute of Biomedicine, Department of \\ Physiology, University of Turku, Turku FI-20520, Finland
}

MIWI catalytic activity is required for spermatogenesis, indicating that piRNA-guided cleavage is critical for germ cell development. To identify meiotic piRNA targets, we augmented the mouse piRNA repertoire by introducing a human meiotic piRNA cluster. This triggered a spermatogenesis defect by inappropriately targeting the piRNA machinery to mouse mRNAs essential for germ cell development. Analysis of such de novo targets revealed a signature for pachytene piRNA target recognition. This enabled identification of both transposable elements and meiotically expressed protein-coding genes as targets of native piRNAs. Cleavage of genic targets began at the pachytene stage and resulted in progressive repression through meiosis, driven at least in part via the ping-pong cycle. Our data support the idea that meiotic piRNA populations must be strongly selected to enable successful spermatogenesis, both driving the response away from essential genes and directing the pathway toward mRNA targets that are regulated by small RNAs in meiotic cells.

[Keywords: MIWI; pachytene piRNAs; RNA cleavage; ping-pong cycle; exogenous piRNAs; spermatogenesis defect] Supplemental material is available for this article.

Received February 16, 2015; revised version accepted April 28, 2015.

Beginning at the pachytene stage of meiosis, the Piwi proteins MIWI and MILI bind a highly abundant and extremely diverse class of piRNAs (Aravin et al. 2006; Girard et al. 2006). These are derived from discrete genomic loci, termed pachytene piRNA clusters, from which emanate an extreme diversity of small RNAs. Nearly all of these map perfectly only to the loci from which they are derived. The importance of pachytene piRNAs is inferred from the essential requirement for their binding partner, MIWI, and its catalytic activity for successful sperm development (Deng and Lin 2002; Reuter et al. 2011).

Despite a clear requirement for MIWI in spermatogenesis, multiple conflicting models exist describing the precise molecular function of MIWI and its piRNA partners. Early proposals contemplated an essential role for MIWI in promoting the expression of a subset of microRNAs (miRNAs) in testes (Grivna et al. 2006a,b). The same studies also reported the association of MIWI with polysomes. This was supported by a separate analysis of Mael mutants that showed that reduced levels of meiotic piRNAs corresponded with a reduction in the translation of some spermiogenic mRNAs (Castañeda et al. 2014). Following consideration of the observation that loading

\footnotetext{
${ }^{5}$ Present address: Institute of Molecular and Cell Biology, Singapore 138673, Singapore

Corresponding author: greg.hannon@cruk.cam.ac.uk

Article is online at http://www.genesdev.org/cgi/doi/10.1101/gad.260455. 115 .
}

of pachytene piRNAs directs MIWI toward the ubiquitin-proteosome pathway for degradation (Zhao et al. 2013), an alternative model emerged, which suggested that in the absence of meiotic piRNAs, MIWI was stabilized so it can inhibit translation. In addition to repressing or activating translation, MIWI has also been implicated in promoting RNA stability (Nishibu et al. 2012; Vourekas et al. 2012). Given that MIWI-associated mRNAs, identified by cross-linking immunoprecipitation (CLIP), were less abundant in Miwi mutants, it was argued that MIWI binding stabilizes bound mRNAs. In this study, MIWI binding did not require meiotic piRNAs but instead must have identified its targets in a way that differs from other Argonaute proteins. Finally, based on the phenotype of Mov10L1 mutants, which lack meiotic piRNAs, MIWI was implicated in promoting DNA damage repair and genome stability, although this could be a direct effect or an indirect consequence of the posited roles of MIWI in gene regulation (Zheng and Wang 2012).

The development of Miwi mutant sperm fails at the round spermatid stage. Mice expressing only a catalytically incompetent MIWI protein have precisely the same

\footnotetext{
(C) 2015 Goh et al. This article is distributed exclusively by Cold Spring Harbor Laboratory Press for the first six months after the full-issue publication date (see http://genesdev.cshlp.org/site/misc/terms.xhtml). After six months, it is available under a Creative Commons License (Attribution-NonCommercial 4.0 International), as described at http:// creativecommons.org/licenses/by-nc/4.0/.
} 
piRNA-directed cleavage regulates spermatogenesis

phenotype, strongly implicating piRNA-directed cleavage as being critical to germ cell development. Based on 5'RACE, LINE1 elements were strongly implicated as direct targets of MIWI (Reuter et al. 2011). This was true despite the fact that meiotic piRNA clusters were initially reported as being depleted of mobile element sequences as compared with the genome as a whole (Girard et al. 2006). However, recent studies have shown that young, potentially active elements are enriched in meiotic piRNA clusters, and these show a bias in orientation such that antisense piRNAs are preferentially produced (Hirano et al. 2014; KA Wasik, OH Tam, I Falciatori, SR Knott, M Hammell, VV Vagin, and GJ Hannon, in prep.). Despite strong evidence for LINEs as pachytene piRNA targets, it is unclear whether LINE activation is the root cause of the sterility of MIWI mutants. Mice lacking a selected piRNA cluster showed a 15-fold elevation in LINE1 expression but were fully fertile (Xu et al. 2008), suggesting that MIWI must also have other targets whose engagement is important for sperm production. We therefore sought to develop strategies to identify such targets so as to help characterize the role of pachytene piRNAs in spermatogenesis.

\section{Results}

Expression of human piRNAs in mouse testes

As a step toward identifying MIWI targets, it was essential to understand the rules that govern piRNA target recognition. To achieve this goal, we sought to produce mice in which we augmented the endogenous piRNA repertoire and then searched for newly acquired cleavage targets. Our hope was that matching these new targets to exogenous piRNAs might reveal rules for piRNA targeting in vivo that would allow us in turn to identify the targets of native piRNAs.

Our strategy was to introduce an entire human piRNA cluster into mice so that its content might contribute to mouse piRNA populations. The sequence of pachytene piRNA clusters is not conserved, yet clusters are found at syntenic locations in mammals (Girard et al. 2006). Given that a major cluster on mouse chromosome 17 (ch17) contributes substantially to mouse piRNA populations, we transferred its $\sim 80-\mathrm{kb}$ human counterpart, found on human ch6, into mice by bacterial artificial chromosome (BAC) transgenesis (termed Hu6 mice) (Fig. 1A).
A

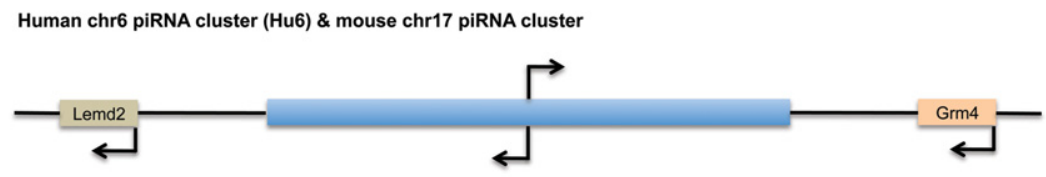

Delta cluster (Hu6 $\Delta$ )

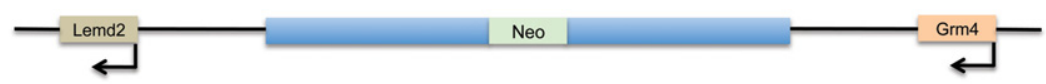

B

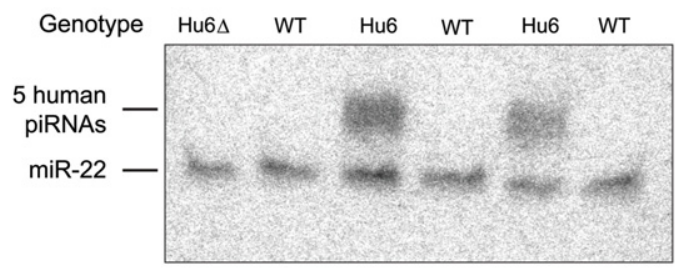

C

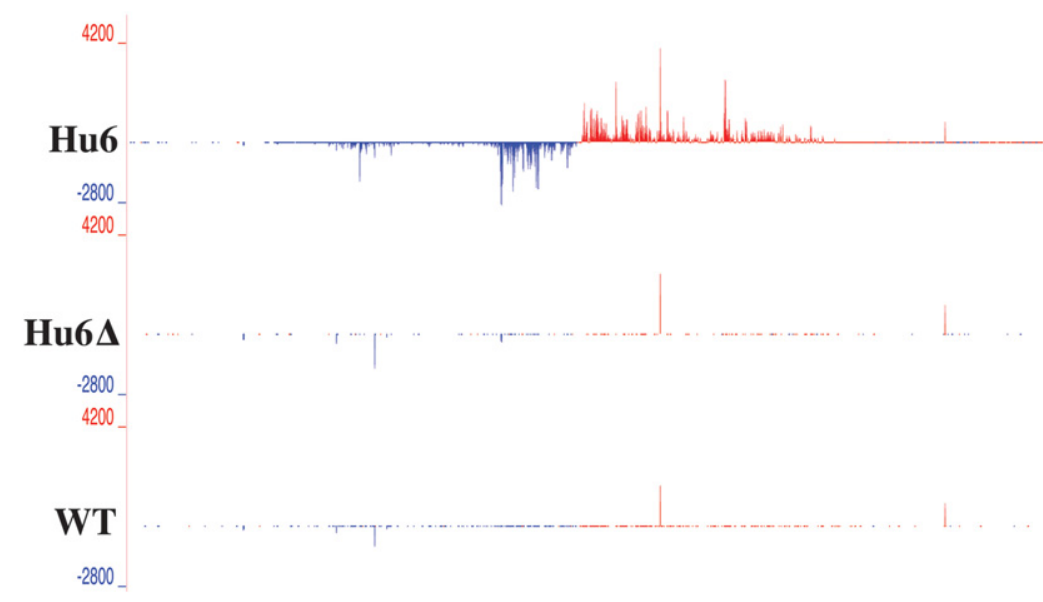

Figure 1. Expression of a human piRNA cluster in mouse testes. $(A$, top $)$ A schematic of the region that contains the bidirectional $\mathrm{Hu} 6 \mathrm{piR}$ NA cluster syntenic to the mouse ch17 cluster is shown. (Bottom) A schematic of the same human region that was engineered to replace the promoter region of the piRNA cluster with a neomycin resistance cassette $(\mathrm{Neo})$ is shown. Arrows indicate transcription start sites for genes and the expected transcription start sites for the piRNA cluster. $(B)$ Northern blotting of human piRNAs. The bands represent the five human piRNAs (30 nucleotides [nt]) and loading control, miR-22 (22 nt), that were blotted in various mouse testis RNA. (C) A genome browser visualization of total small RNAs cloned from Hu6, Hu6s, and wild-type mouse testes and mapped to human ch6 coordinates $33,820,000-33,903,000$. Red and blue signals denote reads that map to the plus and minus strand, respectively, of the genome. $Y$-axis values denote reads per million mapped reads (RPM). 
We also generated mice carrying the human ch6 cluster with an $\sim 4-\mathrm{kb}$ deletion in its central region $(\mathrm{Hu} 6 \Delta)$, presumably removing both transcriptional control elements and the transcription start sites for the divergently synthesized piRNA precursor transcripts (Li et al. 2013). Northern blotting for selected human piRNAs indicated that the cluster was active only in adult mouse testes that contained the intact Hu6 cluster (Fig. 1B). Small RNA sequencing (RNA-seq) of testes total RNA or MIWI immunoprecipitates from transgenic animals also confirmed that piRNAs were produced from the entire human cluster and that these had the characteristic length and first uridine (1U) bias of bona fide primary piRNAs (Fig. 1C; Supplemental Fig. S1).

\section{Human piRNA-guided de novo cleavage patterns reveal a piRNA cleavage signature}

In an effort to identify potential direct cleavage targets of human piRNAs, we performed RNA-seq from sorted Hu6 round spermatids and found that a number of cellular mRNAs were significantly misregulated $(P<0.01)$ (Fig. 2A). We also used global 5'RACE to detect human piRNA-dependent cleavage sites. We initially noticed 5'RACE tags enriched in the Hu6 mouse that corresponded to genes that were also repressed upon introduction of the human piRNA cluster (Fig. 2B,C). By comparing these cleavage sites with piRNAs present only in Hu6 testes, we noted the presence of sense piRNAs that mapped perfectly to the cleaved mRNAs and began precisely at the cleavage site detected by $5^{\prime}$ RACE. This was a strong indication of at least partial operation of the ping-pong cycle in which a cleaved, piRNA-targeted mRNA would be further metabolized to generate a small RNA loaded into a PIWI protein.

This evidence for piRNA-guided cleavage prompted us to search among human ectopic piRNAs for species capable of targeting these mouse mRNAs. By varying the extent of the required complementarity and the number of tolerated mismatches, we found parameters consistent with targeting of detected cleavage sites by human piRNAs but not by any mouse piRNAs (Fig. 2D,E). These parameters indicated a necessity for perfect matching in nucleotides 2-11 (primary seed), with a maximum of four mismatches being tolerated in nucleotides 12-21 (secondary seed). The aggregate of our observations of exogenous piRNA-target pairs permitted us to derive a set of criteria that could be applied to identify targets of native mouse piRNAs. This involved (1) the comparison of 5'RACE tags in Miwi mutants versus heterozygous littermates, (2) the detection of sense piRNAs at cleavage sites that have the size and nucleotide biases of MIWI-bound piRNAs (Supplemental Fig. S2A,B), and (3) the detection of corresponding pachytene piRNAs that meet the mapping parameters described above (see the Materials and Methods for details).

We hypothesized that the piRNA cleavage signature derived from Hu 6 mouse data could be used to identify native piRNA cleavage targets of mouse pachytene piRNAs in wild-type mice. Starting with a list of MIWIdependent 5'RACE tags that are enriched in $\mathrm{Miwi}^{+/-}$ over $\mathrm{Miwi}^{-/-}$spermatogenic cells, we identified and annotated the targets that fulfilled our criteria (Supplemental Fig. S2C,D). Given the vast sequence repertoire of the pachytene piRNA population, it was possible that with the tolerance for four mismatches in the secondary seed, piRNAs could match to identified targets simply by chance. To resolve this issue, we quantified the frequencies of identified antisense piRNAs that match at various secondary seed complementarities to their corresponding targets. We also determined the respective frequencies of randomly selected piRNA secondary seeds, assuming perfect complementarity in the primary seed (see the Materials and Methods). We found that random piRNAs tend to exhibit zero to five matches with identified targets via the secondary seed, which means that antisense piRNAs that complement identified targets with six or more matches do so not simply by chance (Supplemental Fig. S3). Furthermore, we noted that identified antisense piRNAs were also generally better matched to their corresponding targets than the required six secondary seed matches (Supplemental Fig. S3C).

\section{Identification of known piRNA targets validates the piRNA cleavage signature}

As existing data point to transposons and particularly LINEs as targets of the meiotic piRNA pathway (Reuter et al. 2011), we first searched mobile element classes for evidence of piRNA-guided cleavage. We found both active LINE1 families and LTR element families harboring putative piRNA cleavage sites (Supplemental Figs. S2D, S4A,

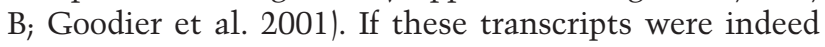
piRNA targets, we expected the loss of MIWI to increase expression of these elements. While TE expression was similar in wild-type and mutant animals at the earlier pachytene spermatocyte stage, by the later round spermatid stage, transposons identified as targets all displayed greater than threefold increases in expression in Miwi mutants (Supplemental Fig. S4C,D). Thus, the parameters that we developed could effectively identify known meiotic piRNA targets.

\section{Meiotic mRNAs as piRNA targets}

Success in identifying transposons as piRNA targets led us to examine the possibility that meiotic piRNAs might also regulate endogenous mRNAs, as predicted by the fact that increased transposon expression alone seemed insufficient to cause arrest at the round spermatid stage (Xu et al. 2008). This was supported by the fact that a sizeable proportion of our piRNA targets was annotated as proteincoding genes (Supplemental Fig. S2D). Using the criteria established above, we identified mRNAs that showed cleavage patterns and corresponding antisense and sense (mRNA-derived) piRNAs consistent with their being targeted directly by MIWI complexes. By the same logic used for validating transposon targets, we expected these mRNAs to be up-regulated in $\mathrm{Miwi}^{-/-}$round spermatids. Compared with randomly selected mRNAs, our putative targets were more likely to be significantly up-regulated 
A

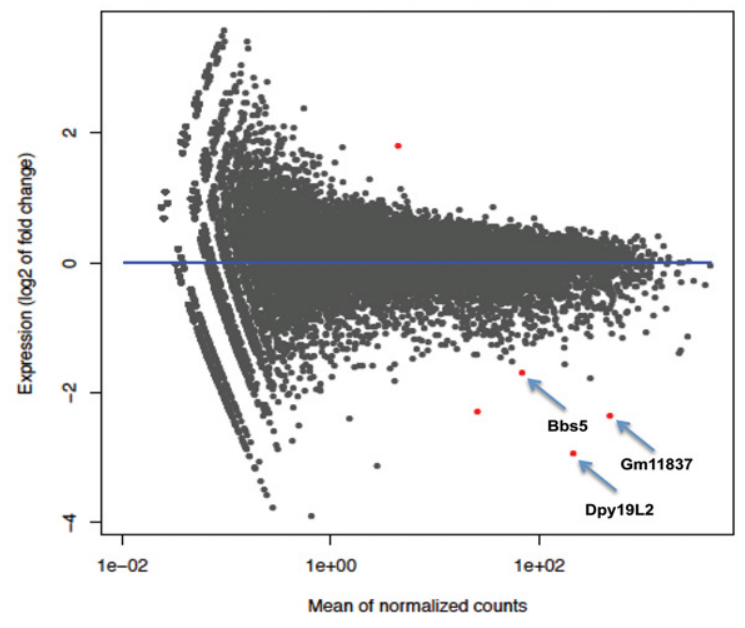

D

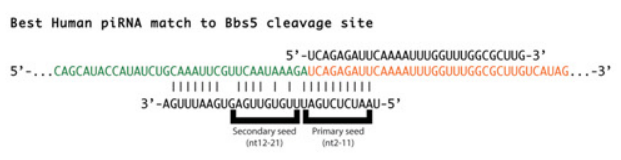

Best Mouse piRNA match to Bbs5 cleavage site

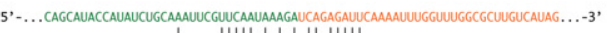

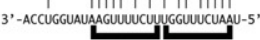

E

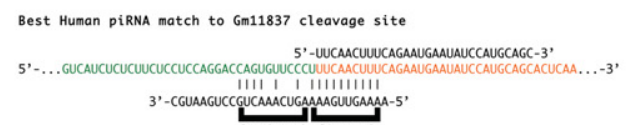

Best Mouse piRNA match to $\mathrm{Gm} 11837$ cleavage site

5 ' -...GUCAUCUCUCUUCUCCUCCAGGACCAGUGUUCCCUUUCAACUUUCAGAUGGAAUAUCCAUGCAGCACUCAA...-3'

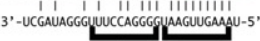

B
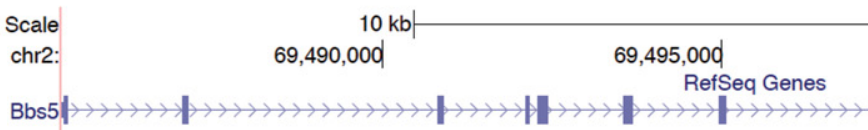

$\mathrm{mm} 9$

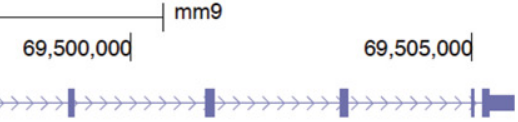

De Novo Cleavage Site

93.4427

Hu6 5'RACE

0.20447

Hu6 sRNA

$-48$
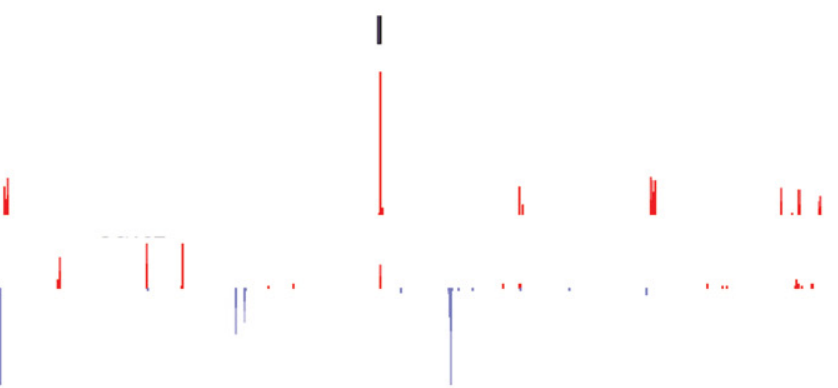

C

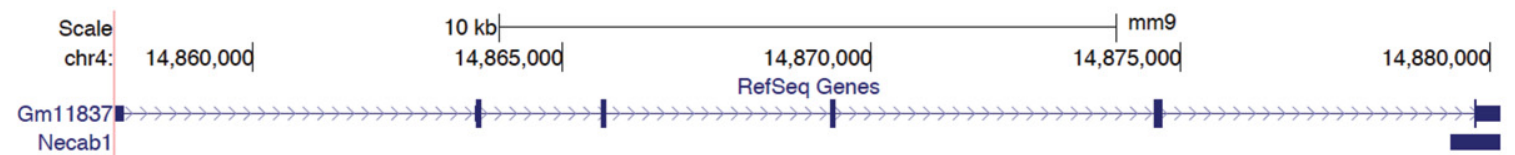

De Novo Cleavage Site
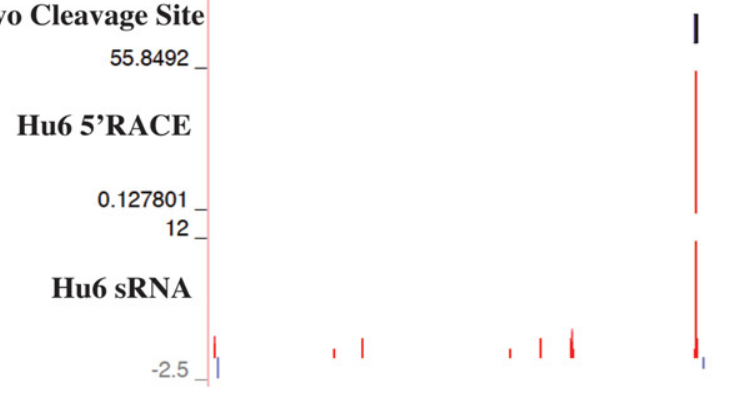

Figure 2. Hu6 human piRNAs target mouse mRNAs for cleavage, thereby revealing a piRNA cleavage signature. $(A)$ A scatter plot depicting the RNA-seq values as sequenced from sorted Hu6 versus wild-type round spermatids. Each dot represents the average RPM of an individual gene. The $X$-axis denotes the mean of average normalized read counts between both wild-type and Hu6 samples, while the $Y$ axis denotes the $\log _{2}$ fold change of RNA levels between Hu6 and wild-type samples. Positive and negative $Y$-axis values denote genes that are up-regulated or down-regulated, respectively, in Hu6 compared with wild-type round spermatids. The red dots denote genes that are significantly misregulated (twofold cutoff). $P<0.01$. The arrows identify three genes of interest. The RNA-seq values are the average of two Hu6 or three wild-type biological replicates. $(B, C)$ A genome browser visualization of round spermatid 5'RACE tags and MIWI-bound small RNA reads sequenced from Hu6 testes, located in mouse Bbs5 (B) or Gm11837 (C). The format is the same as in Figure 1C. (D, E) A schematic showing the targeting of mouse Bbs5 (D) and $G m 11837(E)$ cleavage sites by human piRNAs. The orange sequence denotes the 5'RACE tag enriched in Hu6 round spermatids, and the green sequence denotes the transcript sequence upstream of the cleavage site. The sequence above the 5'RACE tag, if any, depicts a representative Hu6 testis-exclusive sense piRNA that shares a 5'end with the $5^{\prime} \mathrm{RACE}$ tag. The sequence below the 5'RACE tag is the reverse sequence of a representative intergenic cluster guide piRNA. Each vertical bar denotes a perfect base pair between the cleavage site and the guide piRNA. The Hu6 cluster guide piRNA (top) or native mouse guide piRNA (bottom) that can best complement each cleavage site is shown. 
$(P<0.01)$ (Fig. 3A-C, red dots) or were biased to be more highly expressed in $\mathrm{Miwi}^{-1-}$ round spermatids (Fig. 3C, green dots). In total, we identified 330 putative mRNA targets, of which 72 were up-regulated in $\mathrm{Miwi}^{-/-}$round spermatids (Supplemental Tables S1, S2).

Meiotic piRNAs appear roughly at the pachytene stage and build in abundance through the secondary spermatocyte and round spermatid stages. Consistent with this small RNA class regulating endogenous genes, almost all of our proposed mRNA targets of piRNAs showed sharp decreases in wild-type expression as cells progressed from pachytene to round spermatid stages (Fig. 4A, right). Moreover, the degree of differential expression between wild-type and Miwi mutants also increased over this developmental interval (Supplemental Fig. S5A). In contrast, all mRNAs as a class or mRNAs that showed increased expression in Miwi mutants but lacked characteristics of direct targets failed to show this trend (Fig. 4A, left and middle). We note that some of our identified mRNA targets are regulated by the transcription factor $A-M y b$, which also regulates the transcription of Miwi and pachytene piRNA cluster loci (Li et al. 2013). The resulting regulatory interactions fit into a type 1 incoherent feed-forward loop (Supplemental Fig. S5B), which may further reinforce the expression patterns exhibited by these mRNA targets (Alon 2007).

To ask whether targeting by meiotic piRNAs and changes in mRNA levels in Miwi mutants had an effect at the protein level, we performed iTRAQ analyses comparing $\mathrm{Miwi}^{+/-}$and $\mathrm{Miwi}^{-/-}$round spermatids. Out of the subset of proteins encoded by our 72 mRNA targets and detectable by iTRAQ, several showed roughly twofold increases in the absence of MIWI, with TDRD1 standing out as being roughly fourfold increased when piRNA targeting was disrupted (Fig. 4B; Supplemental Fig. S5C,D; Supplemental Table S3).

\section{Pachytene piRNAs silence target mRNAs via} the ping-pong cycle

A key feature of the ping-pong cycle is that fragments of target RNAs form new sense piRNAs, with their $5^{\prime}$ ends coinciding with the PIWI-guided cleavage sites. Although this criterion was applied to define pachytene piRNA targets, the ping-pong cycle is known not to operate robustly in meiotic cells (Reuter et al. 2011; Beyret et al. 2012; Watanabe et al. 2015). We therefore asked whether the sense/antisense piRNA pairs generated from mRNA targets exhibited other characteristics of the ping-pong cycle. In order to regenerate the $1 \mathrm{U}$ in antisense piRNAs, sense piRNAs that undergo the ping-pong cycle tend to have a 10th adenosine (10A) bias (Brennecke et al. 2007; Gunawardane et al. 2007; Wang et al. 2014b). We noted that before filtering for genes identified as direct piRNA targets, all genic sense piRNAs possessed only a $1 \mathrm{U}$ bias (Fig. 5A). If we examined sense piRNAs only from identified protein-coding mRNA targets, we then noticed a slight 10A bias, which was enhanced in the subset of mRNA targets up-regulated in Miwi mutants (Fig. 5B,C). It is important to note that this $10 \mathrm{~A}$ bias is not generated by the $1 \mathrm{U}$ of
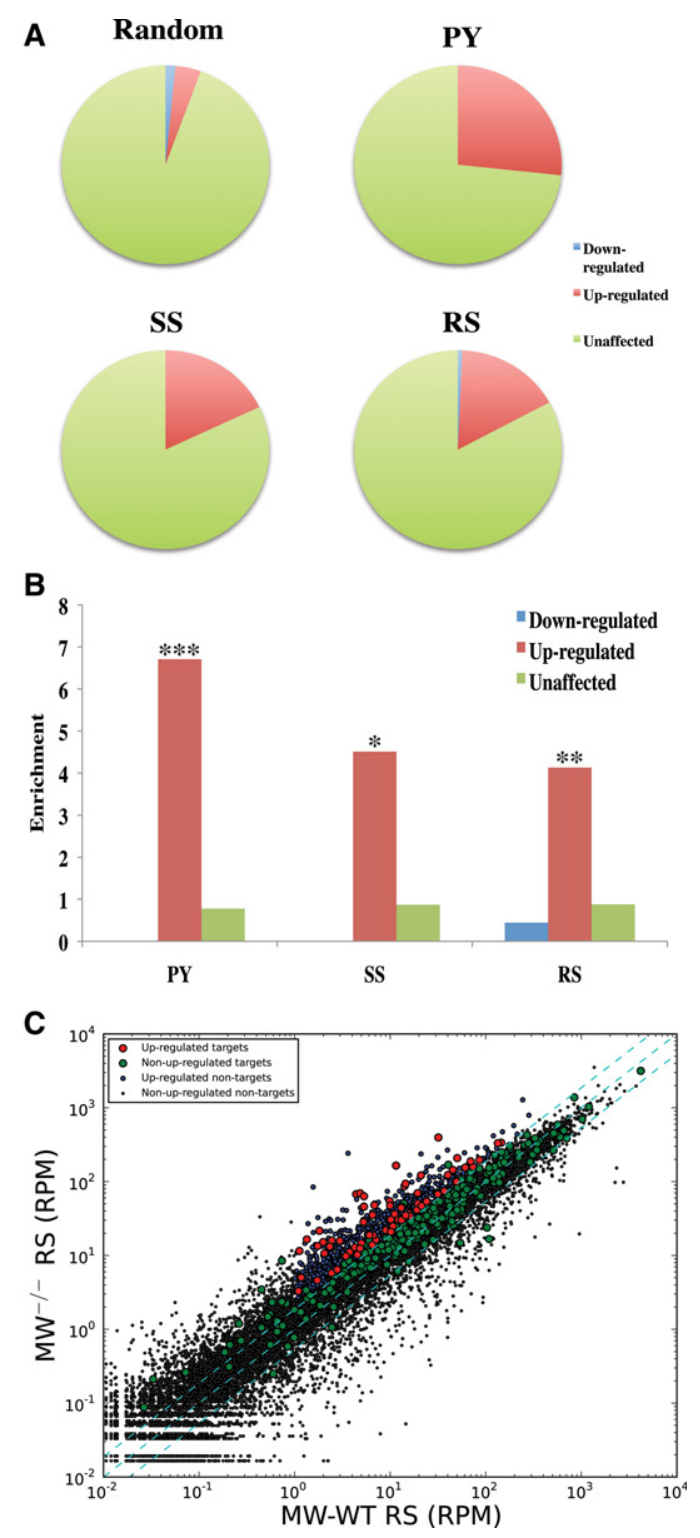

Figure 3. The piRNA cleavage signature identifies meiotic mRNAs as targets of native pachytene piRNAs. (A) The pie charts display proportions of identified mRNA targets, which are significantly misregulated in Miwi knockout versus Miwi wild-type round spermatids (twofold cutoff; $P<0.01$ ) or are not misregulated. "PY," "SS," and "RS" denote the proportions for mRNA targets that were identified separately using 5'RACE tags from sorted pachytene spermatocytes, secondary spermatocytes, or round spermatids, respectively. "Random" denotes the same proportions for mRNAs randomly sampled from mRNAs expressed in round spermatids. $(B)$ A bar graph depicting the enrichment of pie chart proportions in $A$ for various meiotic stages over respective proportions in the "Random" pie chart. $\left({ }^{*}\right) P<3 \times 10^{-6}$; $\left.{ }^{(* *}\right) P<4 \times 10^{-9} ;\left({ }^{* * *}\right) P<7 \times 10^{-26} .(C)$ A scatter plot depicting the RNA-seq values as sequenced from sorted Miwi knockout versus Miwi wild-type round spermatids. Each dot represents the average RPM of an individual gene. The cyan dashed lines (from top left to bottom right) represent a twofold increase cutoff, no fold-change, and a twofold decrease cutoff. The RNA-seq values are the average of five Miwi wild-type or two Miwi knockout biological replicates. 


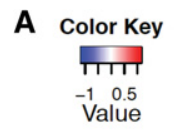

a) Total mRNAs

b)

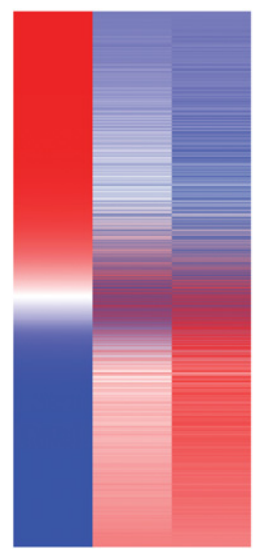

PY SS RS
mRNAs UpRegulated in Miwi-/-

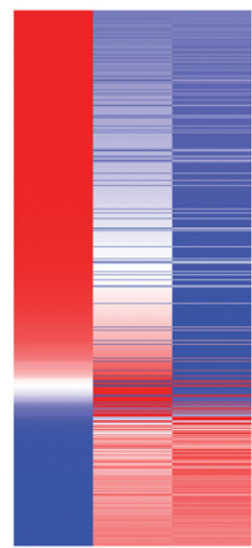

PY SS RS c) mRNA Targets UpRegulated in Miwi-/-

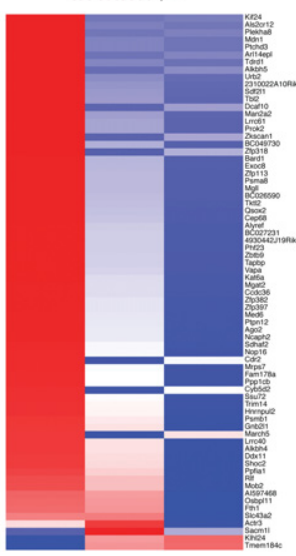

PY SS RS

Figure 4. Pachytene piRNAs silence target mRNAs throughout meiosis. (A) The heat maps of mRNA abundance levels in pachytene spermatocytes (PY), secondary spermatocytes (SS), or round spermatids (RS) sorted from Miwi wild-type testes. Each horizontal row represents an individual mRNA. Red and blue represent higher and lower abundances, respectively, than the row mean. The color intensities represent the $Z$-score from each row mean. Each heat map represents mRNAs with at least 1 RPM in Miwi wildtype round spermatids (panel a), mRNAs significantly up-regulated in $\mathrm{Miwi}^{-/-}$round spermatids (panel $b$ ), and the 72 identified mRNA targets that are significantly up-regulated in $\mathrm{Miwi}^{-/-}$round spermatids (panel $c$ ). The mRNAs in panel $c$ are a subset of those in panel $b$, which in turn are a subset of those in panel $a$. mRNA abundances are calculated from the average of five Miwi wild-type biological replicates. $(B)$ The protein fold change $\left(\mathrm{Miwi}^{-/-}\right.$over $\left.\mathrm{Miwi}^{+/}{ }^{-} ; \mathrm{P}<0.05\right)$ of all proteins that are associated with identified mRNA targets and are detectable by iTRAQ. The error bars denote the standard deviations calculated from the average fold change of two biological replicates.

B

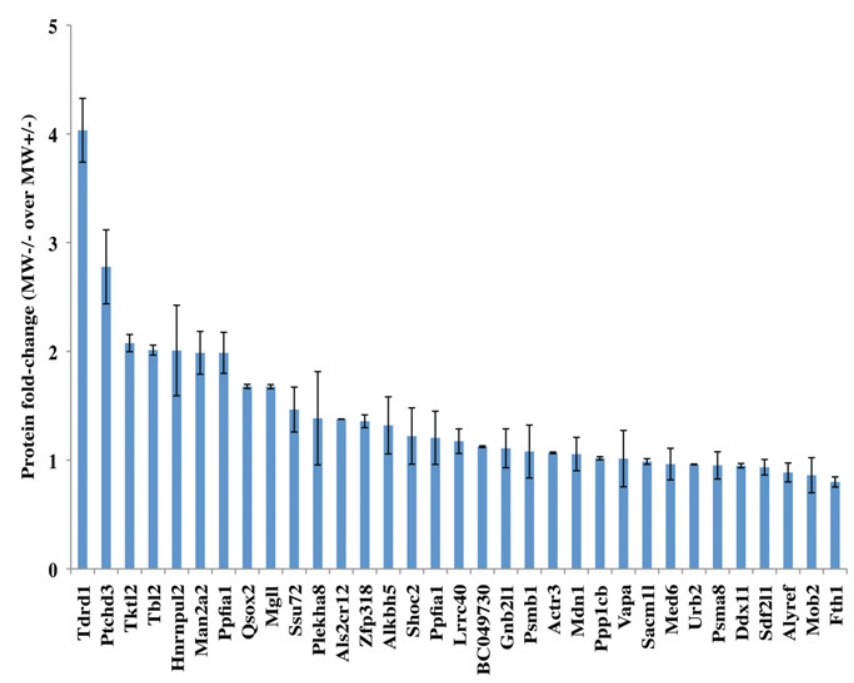

the antisense piRNA, since the first piRNA nucleotide does not base-pair with its target (Reuter et al. 2011; Nakanishi et al. 2012; Wang et al. 2014b). Furthermore, unlike AUB and MILI (Wang et al. 2014b), MIWI does not seem to have a preference to bind targets that have an adenosine 10 nucleotides (nt) downstream from the cleavage site (Supplemental Fig. S6). Therefore, the enrichment of 10A-biased sense piRNAs within identified mRNAs upregulated in Miwi mutants indicates that these sense piRNAs use the ping-pong cycle, leading to the post-transcriptional silencing of their host mRNA.

In flies, the piRNA initiating the cycle often shows a $1 \mathrm{U}$ bias, whereas the piRNA generated in response to cleavage has a 10A bias (Brennecke et al. 2007; Gunawardane et al. 2007). For our targets, both the sense and antisense piRNAs showed a dual 1U/10A bias (Fig. 5C,D). This could reflect a preference for MIWI to bind RNAs with terminal uridines in contrast to flies where AGO3 appears to lack such a preference. Moreover, both sense and antisense piRNAs associate with MIWI, indicating that these meiotic sense/antisense piRNA pairs most likely participate in a homotypic ping-pong cycle.

\section{Augmenting the endogenous pachytene piRNA repertoire causes male sterility}

Previous studies had concluded that the pachytene piRNA sequence is irrelevant to its biological function (Vourekas et al. 2012), although studies reported here and those of others strongly support piRNAs acting in a sequencespecific mode. To probe this issue further, we asked whether alterations in the piRNA repertoire might confer a phenotype. In this regard, two independently derived lines of Hu6 transgenic mice were found to be male sterile (Supplemental Table S4). In contrast, Hu64 mice that failed to produce human piRNAs were fertile. Thus, 


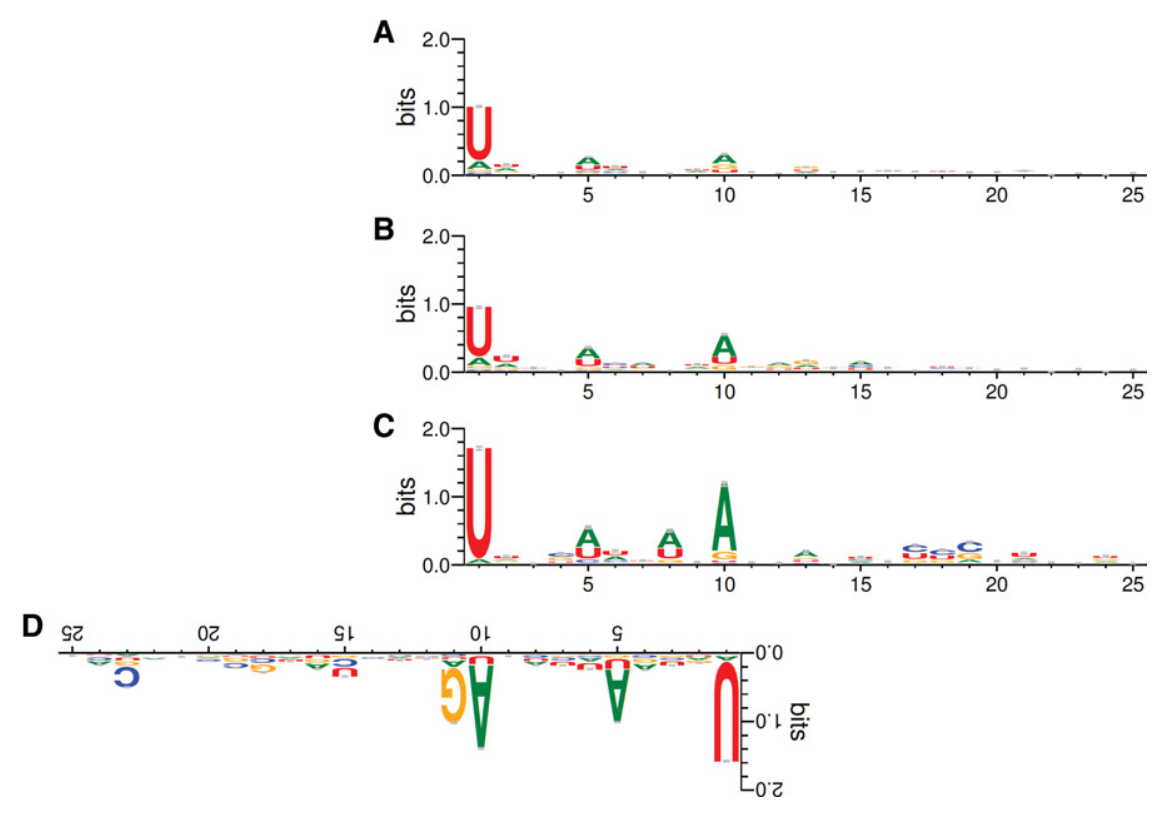

Figure 5. Genic sense piRNAs exhibit the greatest 10A bias when found in $\mathrm{Miwi}^{-/-}$ up-regulated mRNAs that are targeted by antisense guide piRNAs. $(A)$ The nucleotide bias of all genic sense piRNAs that map perfectly to $5^{\prime}$ ends of $5^{\prime}$ RACE tags in mRNAs. These piRNAs derive from mRNAs that satisfy all criteria in the piRNA cleavage signature except that they do not necessarily have a complementary antisense guide piRNA. (B) The nucleotide bias of the subset of $A$ exclusive to $\mathrm{mRNAs}$ that are identified as pachytene piRNA targets. (C) The nucleotide bias of the subset of $B$ exclusive to mRNAs that are significantly up-regulated in $\mathrm{Miwi}^{-/-}$round spermatids. (D) The nucleotide bias of intergenic cluster guide piRNAs that are complementary to the cleavage sites associated with the sense piRNAs in $C$, displayed in reverse orientation. human piRNA production was a likely cause for the infertility of the Hu6 mice.

While Hu6 mice produced sperm, these exhibited an unusual morphology. They had rounded sperm heads as opposed to the hook-shaped heads of normal mouse sperm (Supplemental Fig. S7). This sperm head deformity was suggestive of a defect in sperm acrosome development (Yao et al. 2002; Dam et al. 2006). Acrosome staining revealed that, unlike wild-type sperm, which displayed acrosome staining localized to the outer periphery of their hook-shaped heads, Hu6 sperm displayed mislocalized acrosome staining (Fig. 6A). The acrosome is critical in egg penetration during fertilization, but acrosome defects can be compensated for by intracytoplasmic sperm injection (ICSI) (Yao et al. 2002; Wang et al. 2014a). However, Hu6 sperm still could not fertilize eggs via either in vitro fertilization or ICSI, suggesting that these germ cells have defects more pervasive than those that impact the acrosome (Fig. 6B; Supplemental Table S5). This was supported by high-resolution imaging of mutant sperm, which indicated both defects in acrosome development and incomplete compaction of the Hu6 sperm nuclei (Fig. 6C).

During the extensive chromatin remodeling that occurs in spermiogenesis, most core histones are replaced by transition proteins, which in turn are replaced by protamines, resulting in sperm nuclear compaction (Kimmins and Sassone-Corsi 2005). Therefore, we speculated that the incomplete compaction of Hu6 sperm nuclei was caused by incomplete replacement of core histones with protamines. If this was true, protein quantification should reveal increased levels of core histone variants in Hu6 round spermatids as compared with their wild-type counterparts. Indeed, iTRAQ analysis indicated that among proteins with greater abundance in Hu6 round spermatids, core histone variants formed the major class (Fig. 6D; Supplemental Table S6).
It seemed likely that the observed sperm defects were caused by misregulation of germline genes in Hu6 males. Among those genes down-regulated at both the mRNA and protein levels was Dpy19L2 (Fig. 2A; Supplemental Table S6). Dpy19L2 is noteworthy because Dpy19L2 $2^{-/-}$ mice exhibit male sterility with morphological phenotypes identical to those observed in the Hu6 mice (Pierre et al. 2012; Escoffier et al. 2015; Yassine et al. 2015). Thus, lowered expression of Dpy19L2 was a candidate for a root cause of $\mathrm{Hu} 6$ sterility.

We had initial difficulties in identifying a direct cleavage site for human piRNAs within the mouse Dpy19L2 mRNA. We reasoned that this might be due to incomplete coverage by global 5'RACE libraries prepared from small numbers of sorted cells. We therefore applied a relaxed set of criteria that omitted the $5^{\prime} \mathrm{RACE}$ requirement but relied more on detecting the sense piRNA that is the product of piRNA cleavage. This revealed a cleavage site within the mouse Dpy19L2 mRNA that can be targeted by only a Hu6-specific human piRNA but not any endogenous mouse piRNA (Fig. 7A,B).

In contrast to the mouse gene, the human Dpy19L2 gene (HgDpy19L2) did not contain sequences that were targets of the human piRNA that directed cleavage of the orthologous mouse gene (Fig. 7C). We therefore produced doubly transgenic animals containing the Hu6 cluster and the human Dpy19L2 gene. Not only did the presence of $H g D p y 19 L 2$ rescue the male sterility defect caused by human piRNAs, the doubly transgenic male parent was also able to transmit its $\mathrm{Hu} 6$ cluster and HgDpy19L2 transgenes to its progeny (Fig. 7D).

Given that relaxed target selection criteria allowed us to identify a functionally relevant cleavage site in Dpy19L2, there seems a high likelihood that the 5' RACE libraries prepared from sorted cells were far from saturation. We therefore searched among mouse mRNAs for additional potential cleavage targets that met all of our criteria 
A
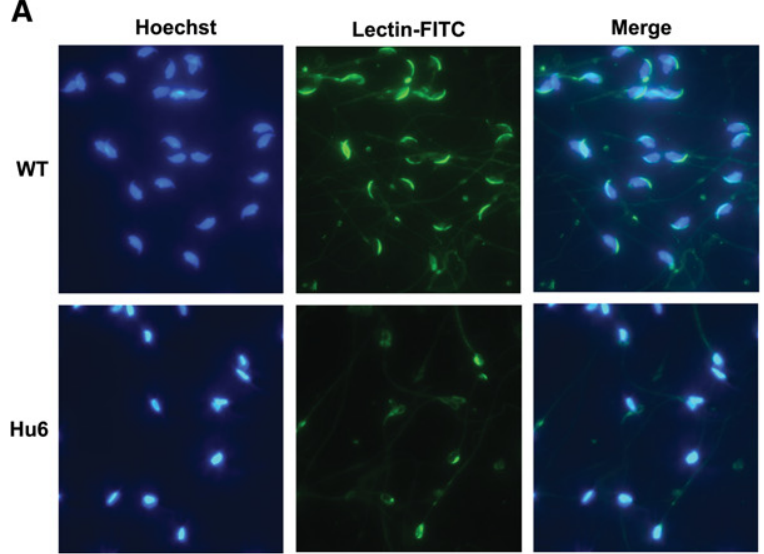

C

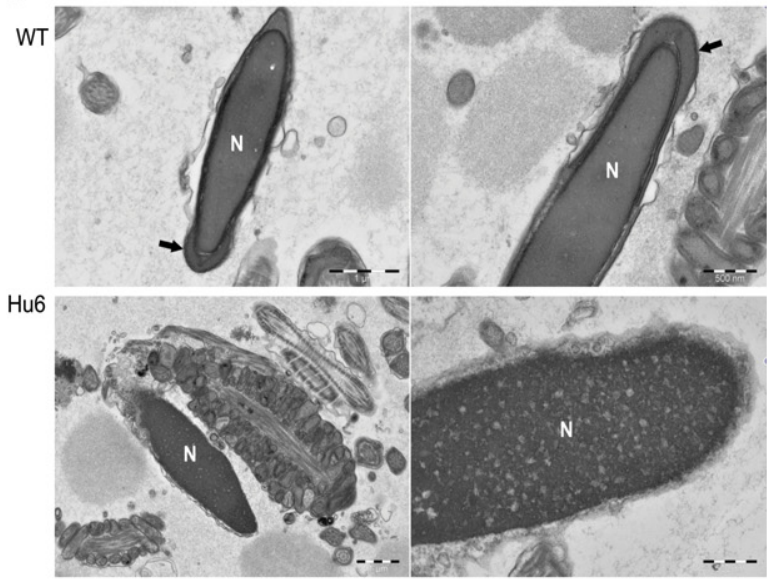

B
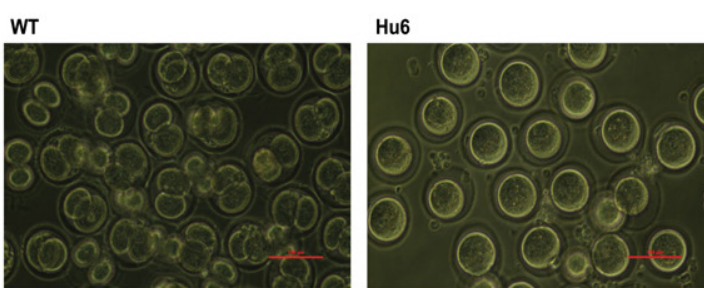

D

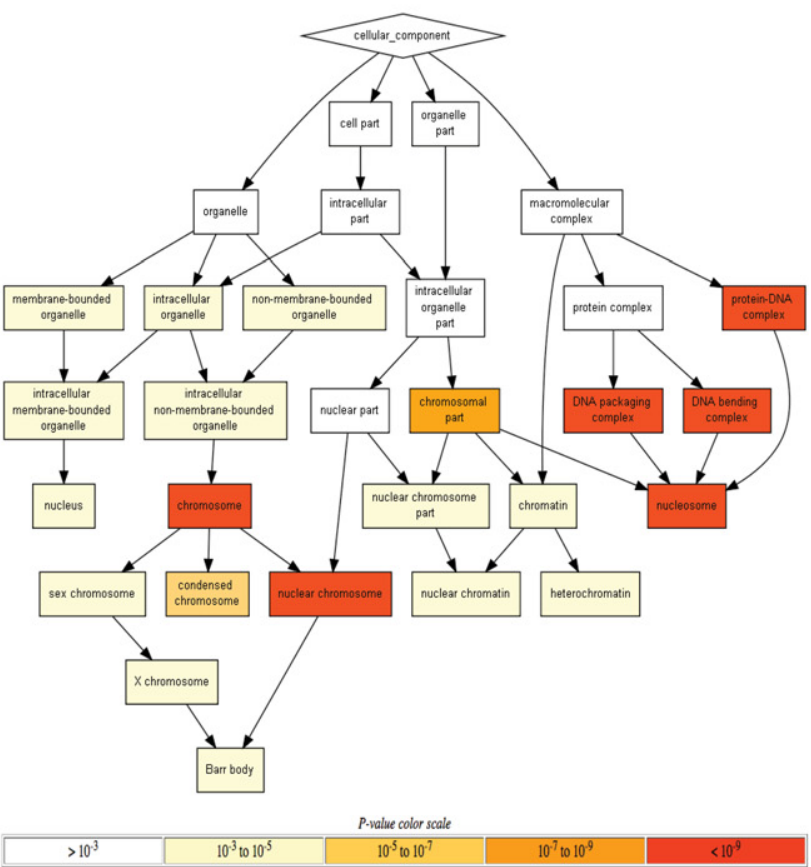

Figure 6. Hu6 males produce defective sperm and are sterile. (A) Representative images of Hoechst and fluorescein isothiocyanate-labeled Pisum sativum agglutinin (FITC-PSA) staining of wild-type (WT) or Hu6 sperm acrosome. (B) Images of eggs $24 \mathrm{~h}$ after fertilization via ICSI with either wild-type or Hu6 sperm. The images are representative of two biological replicates for each genotype. Bar, $100 \mu \mathrm{m}$. $(C)$ Electron microscope image of wild-type (top) and Hu6 (bottom) sperm. Bars: 1eft, $1 \mu \mathrm{m}$; right, $500 \mathrm{~nm}$. (N) Nucleus, (arrow) properly formed acrosome. The images are representative of two biological replicates for each genotype. $(D)$ Gene ontology component analysis of Hu6 round spermatid proteins detected by iTRAQ. The background set is the list of all proteins detected via iTRAQ. The background set was then ranked by protein fold change of Hu6 over wild-type round spermatid, and the top $1 \%$ was use as the input set.

except for a detectable RACE product. This enabled us to identify a list of 628 additional potential targets of native mouse meiotic piRNAs, although each of these must be viewed with less confidence than those having a detectable RACE product (Supplemental Table S7).

\section{Discussion}

\section{An in vivo pachytene piRNA cleavage signature}

Unlike embryonic piRNAs in mammals, meiotic piRNAs have stubbornly resisted efforts to fully understand their functions. These small RNA populations are remarkably diverse and generally match perfectly only to those sites from which they are produced. This negates a simple strategy of searching for targets by matching piRNAs to complementary sequences to which they might direct PIWI proteins to act in trans. Here, we derived an in vivo piRNA cleavage signature for identifying cleavage targets of meiotic piRNAs, which relied on the addition of human piRNAs to the mouse repertoire and the identification of newly acquired cleavage targets among mouse transcripts. The surprising yet most diagnostic feature of this signature is the appearance of target-derived sense piRNAs that map to the $5^{\prime}$ ends of cleaved RNA products. We were able to validate the predictive power of our signature through the identification of known targets of meiotic piRNAs among transposable elements.

\section{Identification of meiotic mRNAs as cleavage targets of pachytene piRNAs}

Through the application of our predictive criteria, we found that a substantial number of mouse mRNAs are targeted by the meiotic piRNA pathway (Supplemental Figs. S8, S9). Consistent with their regulation by piRNAs, these mRNAs 
A

Human piRNA on Mouse Dpy19L2

(

5' - . . A AUGCAUCGGUUCUCCCACAGGACCCUCUUUGGCCUAGCUAUCUUUGTGGGGAUUUUGCACUGGUUACACUU . . . -3, IIIIIIII IIII I IIIIIIIIII GUCCUGGGGAAAACUGUAUCGAUAGAAU

B

Mouse piRNA on Mouse Dpy19L2

5' - . . A AUGCAUCGGUUCUCCCACAGGACCCUCUUUGGCCUAGCUAUCUUUGUGGGGAUUUUGCACUGGUUACACUU . . . - 3' I II IIIIIIIIII AGUCGGUUUCUACCUACGUAUCGAUAGAAU

\section{C}

Same Human piRNA on Human Dpy19L2

5' - . . . GCGCGGCGGUUCUCCAGCAGAACCACUCUCGGCAUCGCUGUCUUUGUGGCAAUUUUACAUUGGUUACAUUU. . . -3' III III II I I III III IIII GUCCUGGGGAAAACUGUAUCGAUAGAAU
D

Parental

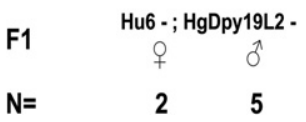

Hu6 - ; HgDpy19L2 $+$

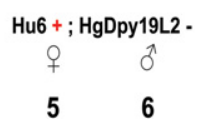

$\mathbf{x}$

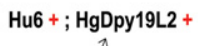

$\begin{array}{cc}\text { Hu6 }+ \text {; } & \text { HgDpy19L2 + } \\ \uparrow & 0 \\ + & 0 \\ 5 & 6\end{array}$

Figure 7. Human piRNA silences mouse Dpy19L2, resulting in male sterility that can be rescued by human Dpy19L2. (A) A schematic showing the targeting of mouse Dpy19L2 mRNA by a Hu6 human piRNA. The format is the same as in Figure 2D, except that the orange sequence denotes the expected cleavage product based on the detected sense piRNA rather than on a 5'RACE tag. (B) A schematic showing the targeting of the mouse Dpy19L2 sequence in $A$ by a mouse piRNA that best matches it. Note that there are eight mismatches within the secondary seed (antisense piRNA nucleotides 12-21). (C) A schematic showing the targeting of the human Dpy19L2 mRNA sequence orthologous to the targeted mouse Dpy19L2 sequence in $A$ by the same Hu6 human piRNA in $A$, with two mismatches in the primary seed. $(D)$ Genetic cross between a doubletransgenic male containing both the Hu6 cluster and human Dpy19L2 gene and a wild-type female. The genotypic frequency of F1 offspring is displayed. Rescue of Hu6 male sterility by $\mathrm{HgDpy} 19 \mathrm{~L} 2$ was observed in at least three double-transgenic males. fell in abundance as wild-type cells progressed from the pachytene spermatocyte to round spermatid stages, and their abundance increased in Miwi mutants. It is tempting to speculate that misexpression of these protein-coding mRNA targets in Miwi mutants might contribute to the arrest at the round spermatid stage. Among the mRNAs regulated by meiotic piRNAs is Tdrd1. Although TDRD1 is essential for cells to successfully complete meiosis, increased expression of TDRD1 and its mislocalization in Miwi mutants could potentially contribute to an arrest at the round spermatid stage (Kojima et al. 2009).

Pachytene piRNA-guided RNA cleavage was first reported through the use of global 5'RACE to identify a cleavage site within a transposon target (Reuter et al. 2011). Our data confirmed this finding and expanded it to include protein-coding mRNAs as piRNA targets. We note that other groups have recently reported observing mRNA silencing by pachytene piRNAs (Gou et al. 2014; Watanabe et al. 2015; Zhang et al. 2015). However, direct cleavage regulation was not demonstrated with 5'RACE in most cases. Also, one report indicates that MIWI and pachytene piRNAs degrade mRNAs in elongated spermatids despite the fact that $\mathrm{Miwi}^{-/-}$germ cells arrest at the earlier round spermatid stage. In this case, the mRNA degradation that they reported appears to be carried out by a MIWI cofactor, CAF1, rather than MIWI itself.

\section{Significance of pachytene piRNAs engaging in the ping-pong cycle}

In the process of identifying native targets of pachytene piRNAs, we noted that MIWI cleavage produced genic sense piRNAs that paired with intergenic antisense piRNAs to undergo a homotypic ping-pong cycle. This was unforeseen given that pachytene piRNAs have long been assumed to avoid such an amplification loop (Reuter et al. 2011; Beyret et al. 2012). This assumption was recently reiterated by a group that reported observing mRNA silencing by pachytene piRNAs (Watanabe et al. 2015). This discrepancy is likely related to their identification of pachytene piRNA targets primarily using a RNA-seq comparison between $\mathrm{Miwi}^{+/-}$and $\mathrm{Miwi}^{-/-}$without filtering for genes that do not possess a piRNA-directed cleavage site. Thus, this strategy has the potential to identify both direct (Fig. 3C, red dots) and indirect (Fig. 3C, blue dots) targets. A recent study had also reported the observation of intergenic antisense piRNAs that target mRNAs and have both a $1 \mathrm{U}$ and $10 \mathrm{~A}$ bias, similar to what we report here (Fig. 5D; Zhang et al. 2015). Given that we see a strong 10A signal only in genic sense piRNAs derived from identified mRNA targets that are up-regulated in Miwi mutants, the ping-pong cycle is likely a functional driving force in the silencing of pachytene piRNA targets.

\section{Sterility caused by the introduction of human piRNAs argues for the sequence-specific function of this small RNA class}

While the observation of piRNA-mRNA ping-pong cycling strongly implies the importance of mRNA silencing to spermatogenesis, it was critical to ask whether altering this silencing process impacted spermatogenesis. Other groups have investigated this question by augmenting the pachytene piRNA repertoire in the presence of wildtype MIWI but failed to see any phenotypic defects (Xu et al. 2008; Watanabe et al. 2015). Here, we found that simply adding a single human piRNA cluster to the mouse piRNA repertoire created profound consequences, 
causing the production of abnormal and completely nonfunctional sperm.

Expression of human piRNAs led to the silencing of a germline gene, Dpy19L2, that is essential for spermiogenesis. The resultant reduction in Dpy19L2 likely caused defects in both acrosome development and nuclear compaction that occur during sperm formation, leading to male sterility. In accord with this hypothesis, introduction of the human ortholog of Dpy19L2 was able to fully rescue the male sterility of $\mathrm{Hu} 6$ mice. Thus, despite the seeming randomness of the highly diverse mouse meiotic piRNAs, there must be stringent evolutionary selection to prevent targeting of mouse genes essential for sperm development.

Just as their abnormal function can negate fertility, sperm development relies on the proper function of native mouse piRNAs. Considered together, our data indicate a closely linked coevolution of meiotic piRNA sequences and protein-coding genes involved in sperm development. Certainly, these sequences must be strongly selected for target avoidance. However, our results indicate that they also evolved to play a role in positively regulating progression into and through spermiogenesis by repressing mRNAs whose inappropriate expression might impair sperm development. Our data support the idea that meiotic piRNA populations must be evolutionarily constrained to enable successful spermatogenesis, both driving the response away from essential genes and directing the pathway toward mRNA targets that are regulated by piRNAs in meiotic cells.

\section{Materials and methods}

\section{piRNA targeting criteria}

An intergenic pachytene piRNA cluster MIWI-immunoprecipitated small RNA $(>28 \mathrm{nt})$ is a guide piRNA that targets a cleavage sequence $\left(-11 \mathrm{nt}\right.$ to $+10 \mathrm{nt}$ of cleavage site) if it first overlaps the $5^{\prime}$ end of the corresponding $5^{\prime} \mathrm{RACE}$ tag in an antisense manner by exactly $10 \mathrm{nt}$, allowing for a mismatch in piRNA nucleotide 1. In addition, it must have perfect complementarity in the primary seed (piRNA nucleotides 2-11) and tolerate at most four mismatches in the secondary seed (piRNA nucleotides 12-21). The complementarity cutoff and seed definitions are based on criteria that allow mouse Bbs5 and Gm11837 to be targeted by only Hu6 human piRNAs but not a single native mouse piRNA and also take into consideration previous in vitro small RNA-based cleavage assays (Reuter et al. 2011; Nakanishi et al. 2012).

\section{piRNA cleavage signature}

A mRNA or transposon was identified as a piRNA-guided cleavage site if it fulfilled these four criteria. First, the mRNA or transposon was not located in previously annotated intergenic pachytene piRNA clusters (Li et al. 2013). Second, the mRNA or transposon contained a $5^{\prime}$ RACE tag where the average $5^{\prime} \mathrm{RACE}$ RPM of $\mathrm{Miwi}^{+/-}$biological replicates was at least $10 \mathrm{RPM}$ and at least 10-fold enriched compared with $\mathrm{Miwi}^{-/-}$biological replicates. Third, there was a MIWI-bound sense piRNA (>28 nt) that mapped perfectly to the $5^{\prime}$ end of the $5^{\prime}$ RACE tag. Fourth, there was a guide piRNA that can target the corresponding cleavage sequence $(-11 \mathrm{nt}$ to $+10 \mathrm{nt}$ of cleavage site). Target identifica- tions were carried out using at least two biological replicates of $5^{\prime}$ RACE data per cell stage per genotype and two biological replicates of MIWI-immunoprecipitated small RNA data. The relaxed cleavage signature was similar to the regular piRNA cleavage signature except that it did not require detection of a 5'RACE tag, and the cleavage site was demarcated by the MIWI-bound sense piRNA.

\section{Transgenic mouse generation}

The RP11-1024C2 human BAC was used to expressed the human Dpy19L2 gene in HgDpy19L2 mice. The RP11-119C22 human BAC was used to express a human ch6 piRNA cluster in Hu6 mice. The BAC used to generate Hu6 6 mice was recombineered from RP11-119C22 by replacing a predicted 4007-base-pair (bp) promoter (as defined by forward, ACCCAACCAATTGTCAGT CAGAAAATGTTTAAATTTACCTATAGCCTGGA; and reverse, GAGGGGAATTTCCTGCCGGCTCTTGCATTCCTCA AAGGTATATACAATTA primer sequences) of the bidirectional human piRNA cluster with a neomycin resistance cassette by Red/ET recombination (Gene Bridges, K002). BACs were extracted from 250-mL bacterial cultures with Nucleobond Xtra Maxi EF (Thermo Fisher Scientific, NC0325254), linearized with PI-SceI (New England Biolabs, R0696), and dialyzed on a 0.025- $\mu \mathrm{m}$ membrane filter (Millipore, VSWP02500) with dialysis buffer (10 mM Tris at $\mathrm{pH} 7.5,0.1 \mathrm{mM}$ EDTA, $100 \mathrm{mM} \mathrm{NaCl}, 10.4$ $\mu \mathrm{g} / \mathrm{mL}$ spermine tetrahydrochloride, $17.8 \mu \mathrm{g} / \mathrm{mL}$ spermidine trihydrochloride). Transgenic animals were generated by pronuclear microinjection of BACs into B6SJL F2 (C57BL/6 × SJL, Jax Laboratory) mouse eggs at the Rodent Genetic Engineering Core at New York University or at the Gene Targeting Shared Resource at Cold Spring Harbor Laboratory. Positive mice were genotyped by PCR. All mice used in our experiments were maintained according to the guidelines of the Cold Spring Harbor Laboratory Institutional Animal Care and Use Committee.

\section{Spermatogenic cell isolation}

Total germ cells were first isolated from adult (2- to 6-mo-old) mouse testes. Pachytene spermatocytes, secondary spermatocytes, and round spermatids were subsequently sorted as described (Bastos et al. 2005), with minor modifications. Briefly, testes were isolated from one adult mouse for each sorting experiment, and the tunica was removed. Tissue was digested for 45 min at $37^{\circ} \mathrm{C}$ in dissociation buffer $(25 \mathrm{mg}$ of collagenase A, 25 $\mathrm{mg}$ of Dispase II, $2.5 \mathrm{mg}$ of DNase I in HBSS). Cells were resuspended at a concentration of $1 \times 10^{6}$ cells per milliliter in $\mathrm{HBSS}^{+}$ (HBSS, $25 \mathrm{mM}$ Hepes, $1.5 \mathrm{mM}$ EDTA, 5\% FBS) and stained with $5 \mathrm{mg} / \mathrm{mL}$ Hoechst (Sigma, 33342) for $30 \mathrm{~min}$ at $37^{\circ} \mathrm{C}$. Immediately before analysis, $1 \mathrm{mg} / \mathrm{mL}$ propidium iodide was added for dead cell exclusion. Analysis was performed in a five-laser AriaII cell sorter (Becton Dickinson). Hoechst was excited with a UV laser at $350 \mathrm{~nm}$, and fluorescence was recorded with a 450/50 filter (Hoechst blue) and 670LP filter (Hoechst red). A 505LP filter was used to separate the emission wavelengths. The three different cell types were gated based on their Hoechst blue-red profile in scatter plots as well as Hoechst blue fluorescence and forward and side scatter properties. After applying gates, cells were collected directly into Trizol-LS for RNA isolation.

\section{Total RNA preparation}

Adult mouse testis tissue or sorted spermatogenic cells were lysed in Trizol or Trizol-LS reagent, and total RNA was isolated according to the manufacturer's recommendations. 


\section{Antibodies}

The rabbit polyclonal antibody against MIWI (MIWI-N3) was produced by injecting the synthesized peptide PTEGDLVGRG RQRGM into two rabbits (Covance, Inc.). The antibody was purified from immunized sera using peptide affinity purification (Thermo Fisher Scientific, 44999). Antibody quality was assessed via Western blotting of testis lysate and immunoprecipitation of $\sim 30$-nt testis small RNAs.

\section{Immunoprecipitation of MIWI-bound small RNAs}

Both mouse testes were homogenized in $1 \mathrm{~mL}$ of lysis buffer (10 $\mathrm{mM}$ Hepes at $\mathrm{pH} 7,100 \mathrm{mM} \mathrm{KCl}, 1.5 \mathrm{mM} \mathrm{MgCl} 2,0.5 \% \mathrm{NP}-40$, $1 \%$ Triton X-100, $10 \%$ glycerol). The lysate was then clarified on a tabletop centrifuge for $30 \mathrm{~min}$, and the supernatant was passed through a $45-\mu \mathrm{m}$ filter. The lysate was incubated with 6 $\mu \mathrm{g}$ of rabbit anti-MIWI-N3 polyclonal antibody and $4 \mathrm{~mL}$ of NT2 buffer $\left(25 \mathrm{mM}\right.$ Tris at $\mathrm{pH} 7.4,150 \mathrm{mM} \mathrm{NaCl}, 1.5 \mathrm{mM} \mathrm{MgCl}_{2}$, $0.05 \% \mathrm{NP}-40$ ) while rotating for $3 \mathrm{~h}$ at $4^{\circ} \mathrm{C}$. One-hundred microliters of protein A-agarose beads (Roche, 11134515001) was prewashed in NT2 buffer and preblocked with 5\% BSA before being incubated with the lysate antibody mixture while rotating for $2 \mathrm{~h}$ at $4^{\circ} \mathrm{C}$. Beads were then washed twice in $1.4 \mathrm{~mL}$ of NT2 buffer, twice in $1.4 \mathrm{~mL}$ of NT-2 (with additional $150 \mathrm{mM}$ $\mathrm{NaCl}$ ), and twice with $1.4 \mathrm{~mL}$ of NT-2 (with additional $350 \mathrm{mM}$ $\mathrm{NaCl}$ ). MIWI-immunoprecipitated small RNAs were eluted by treating beads with $400 \mu \mathrm{g}$ of proteinase K (New England Biolabs, $\mathrm{P} 8107 \mathrm{~S})$ and $1 \times$ proteinase $\mathrm{K}$ buffer (100 $\mathrm{mM}$ Tris at $\mathrm{pH} 7.5,12.5$ mM EDTA, $150 \mathrm{mM} \mathrm{NaCl}, 1 \%$ [w/v] SDS) for $1 \mathrm{~h}$ at $60^{\circ} \mathrm{C}$ with light agitation. Finally, eluted RNA was extracted with phenolchloroform. A quarter of the eluted RNA was visualized with 5' end labeling to verify the precipitation of $\sim 30$-nt RNA species, and the remainder was used for small RNA cloning.

\section{Small RNA cloning}

Total RNA or MIWI-immunoprecipitated RNA (19-33 nt) was first size-selected on a $15 \%$ denaturing polyacrylamide gel. Size-selected small RNAs were then cloned as described (Goh et al. 2014). Libraries were sequenced on an Illumina HiSeq SE36 or SE76 platform.

\section{5'RACE and stranded RNA-seq}

Total RNA from sorted pachytene spermatocytes, secondary spermatocytes, or round spermatids was treated with RQ1 DNase (Promega, M6101) for $30 \mathrm{~min}$ at $37^{\circ} \mathrm{C}$, depleted of ribosomal RNA using the Ribo-Zero kit (Epicentre, MRZH11124), and used to generate global 5'RACE libraries as described (Reuter et al. 2011). Post-amplification libraries were size-selected on a BluePippin 2\% agarose chip (Sage Science, BDF2010) using a cutoff of 180-350 bp. Ribo-depleted RNA was also used for strandspecific RNA libraries, which were constructed using the ScriptSeq version 2 RNA-seq library preparation kit (Epicentre, SSV21124). Both global 5'RACE and ScriptSeq libraries were sequenced on an Illumina HiSeq SE76 platform.

\section{Northern blotting}

Total RNA was separated on a $15 \%$ denaturing polyacrylamide gel. RNA was then transferred from the gel onto a Hybond ${ }^{+}$membrane in $0.5 \times \mathrm{TBE}$ at $20 \mathrm{~V}$ for $90 \mathrm{~min}$. The membrane was UV cross-linked at $1200 \mu \mathrm{J} / \mathrm{cm}^{2}$ and then hybridized in Ultrahyb hybridization buffer for $1 \mathrm{~h}$. We used five different DNA oligonucleotides for Northern blotting: GGCCTCACGCTCCTGATCTG
TAAAA, TATCATGCAGTTGTATTCCATTGTA, AAAGACG TTTCССТTTCAACTCTA, TACTAATCTATCTTCCСAACA TCA, and CATCCATCTAAAGTCTCTCCAAATA. These oligonucleotides are complementary to five different human piRNAs in the Hu6 piRNA cluster, as determined from previous small RNA-seq data of human testes (Girard et al. 2006). The Northern blotting oligonucleotides were 5 '-labeled, pooled, and hybridized to the membrane overnight. The membrane was then washed twice with $2 \times$ SSC and $0.1 \%$ SDS and twice with $1 \times$ SSC and $0.1 \%$ SDS. The washed membrane was exposed onto a phosphoplate overnight before being viewed on a Fuji FLA-5000 PhosphorImager. All hybridization and wash steps were carried out at $37^{\circ} \mathrm{C}$.

\section{Determination of sterility}

Sexually mature males (2-6 mo old) were crossed with CD-1 females. Males are considered sterile if no pups were born after at least 2 mo of crossing.

\section{Preparation of paraffin-embedded testis sections for microscopy}

Mouse testes were extracted from adult mice and fixed in Bouin's solution (Sigma, HT10132) overnight at $4^{\circ} \mathrm{C}$ and then washed for $30 \mathrm{~min}$ twice in $50 \%$ ethanol and twice in $70 \%$ ethanol. After fixation, testis samples were embedded in OCT blocks and sectioned to produce $6-\mu \mathrm{m}$ sections that were separated by $30 \mu \mathrm{m}$. Slides were stained with hematoxylin and eosin, and images were acquired with a Nikon Eclipse Ti microscope.

Staining of sperm acrosome by fluorescein isothiocyanatelabeled Pisum sativum agglutinin (FITC-PSA)

Mouse cauda epididymis was isolated and dissected in $1 \times$ DPBS. Sperm were counted, and 15,000 sperm were spotted onto a slide, permeabilized with methanol, and then stained with FITC-PSA as described (Mendoza et al. 1992) and subsequently with Hoechst (Sigma, H33342). Images were acquired with the Nikon Eclipse Ti microscope.

\section{ITRAQ}

Sorted round spermatids were pelleted, frozen in liquid nitrogen, and then sent to the Proteomics Shared Resources at Cold Spring Harbor Laboratory for protein extraction and quantification via iTRAQ, which was performed as described (Scuoppo et al. 2012).

\section{Acknowledgments}

We thank P. Zamore, C. Tipping, and G. Farley for the gift of $\mathrm{Miwi}^{-1-}$ mice; S.Y. Kim for assisting in generating the HgDpy19L2, Hu6, and Hu6 $\Delta$ transgenic mice and assisting in IVF and ICSI; R. Pillai and M. Reuter for advice on global 5'RACE library preparation; E. Lee, E. Hodges, and E. Ghiban for handling library sequencing; S. Boettcher for managing laboratory equipment and reagents; M. Mosquera for mouse genotyping; M. Cahn, J. Coblentz, G. Munoz, and L. Bianco for mouse husbandry; D. Pappin for assisting in iTRAQ; and C. Carlston, J. Ipsaro, V. Vagin, C. Hammell, M. Timmermans, and V. Ambros for helpful discussions. This work was supported by the National Institutes of Health R37 grant GM062534-14 to G. J.H. iTRAQ was performed with assistance from the Cold Spring Harbor Laboratory Proteomics Shared Resource, which is supported by Cancer Center support grant 5P30CA045508. W.S.S. G. is a McClintock Fellow of the Watson School of Biological 
Sciences and is supported by the NSS Scholarship from the Agency for Science, Technology and Research, Singapore. O.H.T. is supported by a fellowship of the Human Frontier Science Program. R.B. is supported by the Starr Centennial Scholarship from the Watson School of Biological Sciences. G.J.H. is a Howard Hughes Medical Institute Investigator.

\section{References}

Alon U. 2007. Network motifs: theory and experimental approaches. Nat Rev Genet 8: 450-461.

Aravin A, Gaidatzis D, Pfeffer S, Lagos-Quintana M, Landgraf P, Iovino N, Morris P, Brownstein MJ, Kuramochi-Miyagawa S, Nakano T, et al. 2006. A novel class of small RNAs bind to MILI protein in mouse testes. Nature 442: 203-207.

Bastos H, Lassalle B, Chicheportiche A, Riou L, Testart J, Allemand I, Fouchet P. 2005. Flow cytometric characterization of viable meiotic and postmeiotic cells by Hoechst 33342 in mouse spermatogenesis. Cytometry 65A: 40-49.

Beyret E, Liu N, Lin H. 2012. piRNA biogenesis during adult spermatogenesis in mice is independent of the ping-pong mechanism. Cell Res 22: 1429-1439.

Brennecke J, Aravin AA, Stark A, Dus M, Kellis M, Sachidanandam R, Hannon GJ. 2007. Discrete small RNA-generating loci as master regulators of transposon activity in Drosophila. Cell 128: 1089-1103.

Castañeda J, Genzor P, van der Heijden GW, Sarkeshik A, Yates JR, Ingolia NT, Bortvin A. 2014. Reduced pachytene piRNAs and translation underlie spermiogenic arrest in Maelstrom mutant mice. EMBO J 33: 1999-2019.

Dam AHDM, Feenstra I, Westphal JR, Ramos L, van Golde RJT, Kremer JAM. 2006. Globozoospermia revisited. Hum Reprod Update 13: 63-75.

Deng W, Lin H. 2002. miwi, a murine homolog of piwi, encodes a cytoplasmic protein essential for spermatogenesis. Dev Cell 2: 819-830.

Escoffier J, Yassine S, Lee HC, Martinez G, Delaroche J, Coutton C, Karaouzène T, Zouari R, Metzler-Guillemain C, PernetGallay K, et al. 2015. Subcellular localization of phospholipase $\mathrm{C} \zeta$ in human sperm and its absence in DPY19L2-deficient sperm are consistent with its role in oocyte activation. Mol Hum Reprod 21: 157-168.

Girard A, Sachidanandam R, Hannon GJ, Carmell MA. 2006. A germline-specific class of small RNAs binds mammalian Piwi proteins. Nature 442: 199-202.

Goh WSS, Seah JWE, Harrison EJ, Chen C, Hammell CM, Hannon GJ. 2014. A genome-wide RNAi screen identifies factors required for distinct stages of $C$. elegans piRNA biogenesis. Genes Dev 28: 797-807.

Goodier JL, Ostertag EM, Du K, Kazazian HH. 2001. A novel active L1 retrotransposon subfamily in the mouse. Genome Res 11: 1677-1685.

Gou L-T, Dai P, Yang J-H, Xue Y, Hu Y-P, Zhou Y, Kang J-Y, Wang X, Li H, Hua M-M, et al. 2014. Pachytene piRNAs instruct massive mRNA elimination during late spermiogenesis. Cell Res 24: 680-700.

Grivna ST, Beyret E, Wang Z, Lin H. 2006a. A novel class of small RNAs in mouse spermatogenic cells. Genes Dev 20: 1709-1714.

Grivna ST, Pyhtila B, Lin H. 2006b. MIWI associates with translational machinery and PIWI-interacting RNAs (piRNAs) in regulating spermatogenesis. Proc Natl Acad Sci 103: 13415-13420.

Gunawardane LS, Saito K, Nishida KM, Miyoshi K, Kawamura Y, Nagami T, Siomi H, Siomi MC. 2007. A slicer-mediated mechanism for repeat-associated siRNA $5^{\prime}$ end formation in Drosophila. Science 315: 1587-1590.

Hirano T, Iwasaki YW, Lin ZYC, Imamura M, Seki NM, Sasaki E, Saito K, Okano H, Siomi MC, Siomi H. 2014. Small RNA profiling and characterization of piRNA clusters in the adult testes of the common marmoset, a model primate. RNA 20: 1223-1237.

Kimmins S, Sassone-Corsi P. 2005. Chromatin remodelling and epigenetic features of germ cells. Nature 434: 583-589.

Kojima K, Kuramochi-Miyagawa S, Chuma S, Tanaka T, Nakatsuji N, Kimura T, Nakano T. 2009. Associations between PIWI proteins and TDRD1/MTR-1 are critical for integrated subcellular localization in murine male germ cells. Genes Cells 14: 1155-1165.

Li XZ, Roy CK, Dong X, Bolcun-Filas E, Wang J, Han BW, Xu J, Moore MJ, Schimenti JC, Weng Z, et al. 2013. An ancient transcription factor initiates the burst of piRNA production during early meiosis in mouse testes. Mol Cell 50: 67-81.

Mendoza C, Carreras A, Moos J, Tesarik J. 1992. Distinction between true acrosome reaction and degenerative acrosome loss by a one-step staining method using pisum sativum agglutinin. J Reprod Fertil 95: 755-763.

Nakanishi K, Weinberg DE, Bartel DP, Patel DJ. 2012. Structure of yeast Argonaute with guide RNA. Nature 486: 368 374.

Nishibu T, Hayashida Y, Tani S, Kurono S, Kojima-Kita K, Ukekawa R, Kurokawa T, Kuramochi-Miyagawa S, Nakano T, Inoue $\mathrm{K}$, et al. 2012. Identification of MIWI-associated poly (A) RNAs by immunoprecipitation with an anti-MIWI monoclonal antibody. Biosci Trends 6: 248-261.

Pierre V, Martinez G, Coutton C, Delaroche J, Yassine S, Novella C, Pernet-Gallay K, Hennebicq S, Ray PF, Arnoult C. 2012. Absence of Dpy1912, a new inner nuclear membrane protein, causes globozoospermia in mice by preventing the anchoring of the acrosome to the nucleus. Development 139: 2955-2965.

Reuter M, Berninger P, Chuma S, Shah H, Hosokawa M, Funaya C, Antony C, Sachidanandam R, Pillai RS. 2011. Miwi catalysis is required for piRNA amplification-independent LINE1 transposon silencing. Nature 480: 264-267.

Scuoppo C, Miething C, Lindqvist L, Reyes J, Ruse C, Appelmann I, Yoon S, Krasnitz A, Teruya-Feldstein J, Pappin D, et al. 2012. A tumour suppressor network relying on the polyaminehypusine axis. Nature 487: 244-248.

Vourekas A, Zheng Q, Alexiou P, Maragkakis M, Kirino Y, Gregory BD, Mourelatos Z. 2012. Mili and Miwi target RNA repertoire reveals piRNA biogenesis and function of Miwi in spermiogenesis. Nat Struct Mol Biol 19: 773-781.

Wang H, Wan H, Li X, Liu W, Chen Q, Wang Y, Yang L, Tang H, Zhang X, Duan E, et al. 2014a. Atg7 is required for acrosome biogenesis during spermatogenesis in mice. Cell Res 24: 852-869.

Wang W, Yoshikawa M, Han BW, Izumi N, Tomari Y, Weng Z, Zamore PD. 2014b. The initial uridine of primary piRNAs does not create the tenth adenine that is the hallmark of secondary piRNAs. Mol Cell 56: 708-716.

Watanabe T, Cheng E-C, Zhong M, Lin H. 2015. Retrotransposons and pseudogenes regulate mRNAs and lncRNAs via the piRNA pathway in the germline. Genome Res 25: 368-380.

Xu M, You Y, Hunsicker P, Hori T, Small C, Griswold MD, Hecht NB. 2008. Mice deficient for a small cluster of Piwi-interacting RNAs implicate Piwi-interacting RNAs in transposon control. Biol Reprod 79: 51-57. 
Goh et al.

Yao R, Ito C, Natsume Y, Sugitani Y, Yamanaka H, Kuretake S, Yanagida K, Sato A, Toshimori K, Noda T. 2002. Lack of acrosome formation in mice lacking a Golgi protein, GOPC. Proc Natl Acad Sci 99: 11211-11216.

Yassine S, Escoffier J, Martinez G, Coutton C, Karaouzène T, Zouari R, Ravanat J-L, Metzler-Guillemain C, Lee HC, Fissore $\mathrm{R}$, et al. 2015. Dpy1912-deficient globozoospermic sperm display altered genome packaging and DNA damage that compromises the initiation of embryo development. Mol Hum Reprod 21: 169-185.
Zhang P, Kang J-Y, Gou L-T, Wang J, Xue Y, Skogerboe G, Dai P, Huang D-W, Chen R, Fu X-D, et al. 2015. MIWI and piRNAmediated cleavage of messenger RNAs in mouse testes. Cell Res 25: 193-207.

Zhao S, Gou L-T, Zhang M, Zu L-D, Hua M-M, Hua Y, Shi H-J, Li Y, LiJ, LiD, et al. 2013. piRNA-triggered MIWI ubiquitination and removal by APC/C in late spermatogenesis. Dev Cell24:13-25.

Zheng K, Wang PJ. 2012. Blockade of pachytene piRNA biogenesis reveals a novel requirement for maintaining post-meiotic germline genome integrity. PLoS Genet 8: e1003038. 


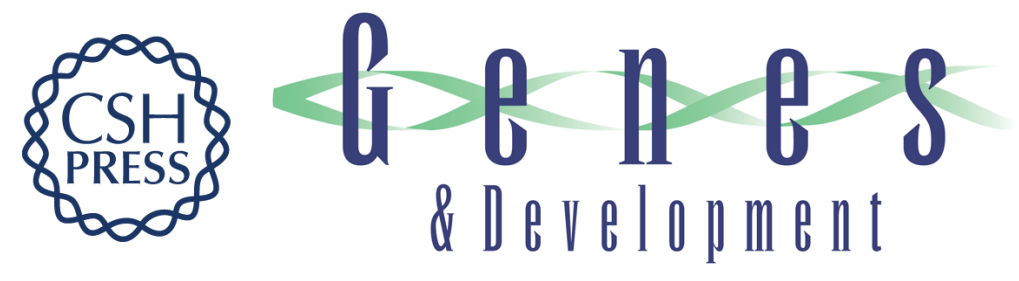

\section{piRNA-directed cleavage of meiotic transcripts regulates spermatogenesis}

Wee Siong Sho Goh, Ilaria Falciatori, Oliver H. Tam, et al.

Genes Dev. 2015, 29:

Access the most recent version at doi:10.1101/gad.260455.115

\section{Supplemental http://genesdev.cshlp.org/content/suppl/2015/05/19/29.10.1032.DC1 Material}

References This article cites 36 articles, 11 of which can be accessed free at: http://genesdev.cshlp.org/content/29/10/1032.full.html\#ref-list-1

Creative This article is distributed exclusively by Cold Spring Harbor Laboratory Press for the first Commons six months after the full-issue publication date (see

License http://genesdev.cshlp.org/site/misc/terms.xhtml). After six months, it is available under a Creative Commons License (Attribution-NonCommercial 4.0 International), as described at http://creativecommons.org/licenses/by-nc/4.0/.

Email Alerting Receive free email alerts when new articles cite this article - sign up in the box at the top Service right corner of the article or click here.

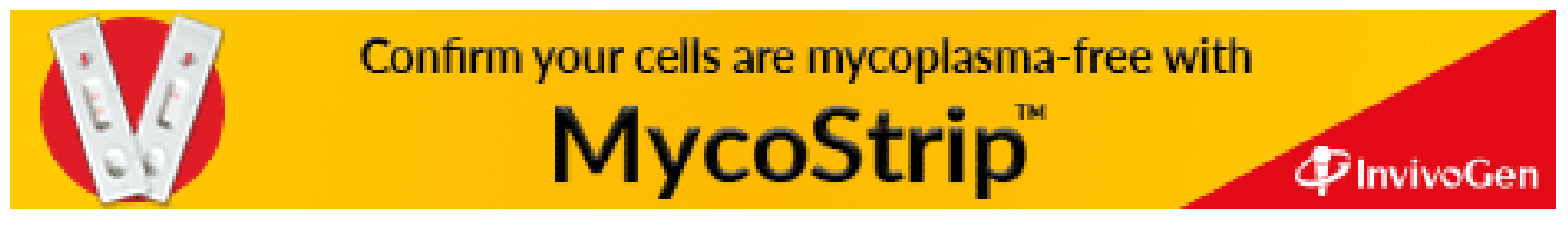

